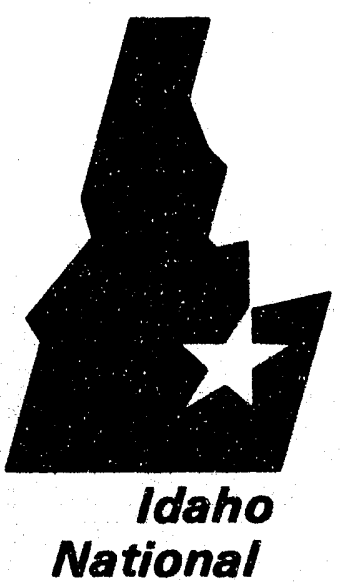

Engineering

Laboratory

Managed

by the U.S.

Department

of Energy
EGG-SARE- 10463

September 1992

\section{TWO-STAGE SAMPLING FOR} ACCEPTANCE TESTING

Corwin L. Atwood

Mark F. Bryan $\stackrel{n}{\infty} \operatorname{Erg} G_{\text {idaio }}$

Work performed under No. DE-ACO7-761DO1570 


\section{DISCLAIMER}

This book wes prepared as an account of work sponsored by an agency of the United States Government. Neither the United States Government nor any agency thereof. nor any of their employees. makes any warranty, express or implied, or assumes any legal liability or responsibility for the accuracy. completeness, or usefulness of any information, apparatus, product or process disclosed, or represents that its use would not infringe privately owned rights. References herein to any specific commercial product, process, or service by trade name, trademerk, manufacturer, or otherwise. does not necessarily constitute or imply its endorsement, recommendation, or favorit. $f$ by the United States Government or any agency thereof. The views and odinions of authors expressed harein do not necessarily state or reflect those of the United States Government or any agency thereot. 
EGG-SARE--10463

DE93 005234

\title{
Two-Stage Sampling for Acceptance Testing
}

Corwin L. Atwood and Mark F. Bryan

September 1992

\author{
Idaho National Engineering Laboratory \\ EG\&G Idaho, Inc. \\ Idaho Falls, ID 83415
}

Work supported by the U. S. Department of Energy

Under DOE Field Office, Idaho

Contract DE-AC07-76ID01570 


\section{ABSTRACT}

Sometimes a regulatory requirement or a quality-assurance procedure sets an allowed maximum on a confidence limit for a mean. If the sample mean of the measurements is below the allowed maximum, but the confidence limit is above it, a very widespread practice is to increase the sample size and recalculate the confidence bound. The confidence level of this two-stage procedure is rarely found correctly, but instead is typically taken to be the numinal confidence level, found as if the final sample size had been specified in advance. In typical settings, the correct nominal $\alpha$ should be between the desired $P$ (Type I error) and half that value. This note gives tables for the correct $\alpha$ to use, some plots of power curves, and an example of correct two-stage sampling. 


\section{CONTENTS}

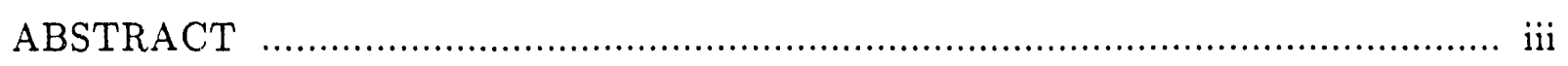

1 BACKGROUND .................................................................................. 1

1.1 The Procedure Considered ................................................................. 1

1.2 Related Literature .......................................................................... 2

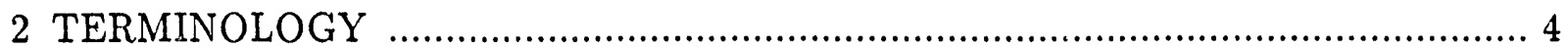

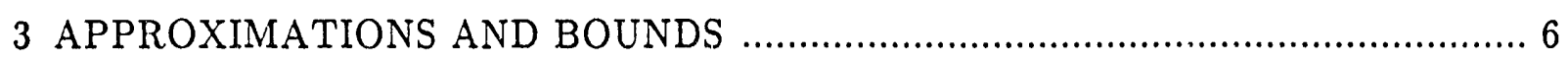

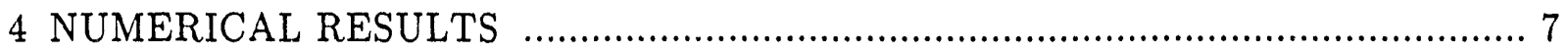

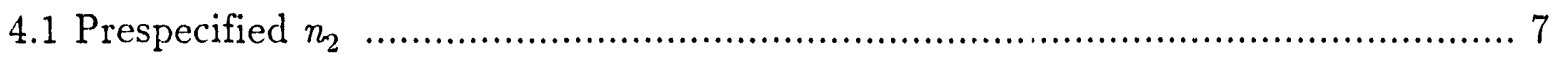

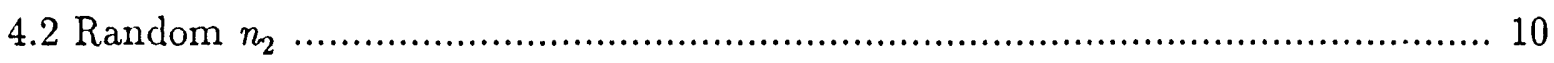

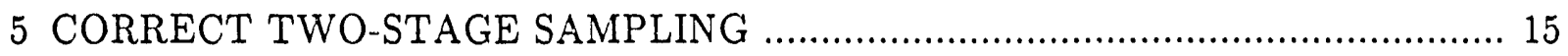

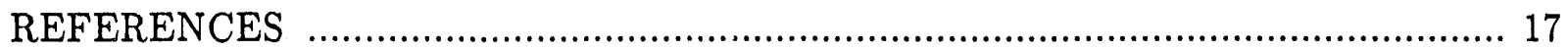

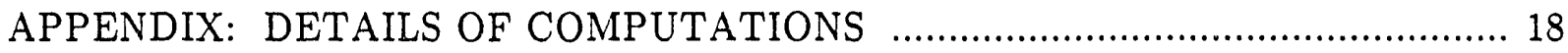




\section{TWO-STAGE SAMPLING FOR ACCEPTANCE TESTING}

\section{BACKGROUND}

\subsection{The Procedure Considered}

Sometimes a regulatory requirement or a quality-assurance procedure sets an allowed maximum on a confidence bound for a mean. It may be that measurements are expensive, so a small sample size is desirable, but that failing to meet the requirement is even more expensive. In such cases, the following practice seems to be widespread: if the sample mean of the measur nents is below the allowed maximum, but the confidence bound is above it, increase the sample size and recalculate the confidence bound. Calculate the second confidence bound as if the final sample size had been specified in advance. This calculation "as if" is incorrect because it does not account for the twostage nature of the sampling, but this fact is generally ignored.

For example, the EPA(1986) guide SW-846 (pp. nine-14 through nine-17) gives a hypothetical example in which preliminary information suggests that six sludge samples should be sufficient to show that the $90 \%$ upper confidence limit (UCL) for barium concentration is less than the regulatory limit. To be safe, nine sludge samples are collected, but only six are initially analyzed. Based on these initial analyses, the sample mean is less than the regulatory limit, but the $90 \% \mathrm{UCL}$ is above the regulatory limit. The values are

$$
\begin{aligned}
& \bar{x}_{1}=94.67 \mathrm{ppm} \\
& s_{1}=9.52 \mathrm{ppm} \\
& \mathrm{UCL}=\bar{x}_{1}+t_{0.90}(5) s_{1} / \sqrt{6}=100.41 \mathrm{ppm} \\
& \text { regulatory limit }=100 \mathrm{ppm}
\end{aligned}
$$

Therefore, the remaining three samples are analyzed; actually, the UCL is so close to the regulatory limit that only one more analysis may be necessary, but all three are analyzed to be safe. This yields 
$\bar{x}=93.56 \mathrm{ppm}$

$s=7.75 \mathrm{ppm}$

$\mathrm{UCL}=\bar{x}+t_{0.90}(8) s / \sqrt{9}=97.16 \mathrm{ppm}$.

Here the UCL is based on all nine measurements, calculated as if the sample size were initially fixed at nine. Because it is less than the regulatory limit, SW-846 states, "it is definitively concluded that barium is not present in the sludge at a hazardous level." This argument is too simple, because it ignores the two-stage sampling.

Since we became interested in this issue several years ago, we have encountered similar two-stage methods in other contexts: (1) Oil samples from many electrical transformers were analyzed to determine which transformers had unacceptable levels of PCB. If the PCB level was too high, the transformer had to be cleaned or disposed of, either process an expensive one. (2) In a different project, millions of particles were manufactured in a single batch. Samples of the particles were measured in about two dozen ways to satisfy the many quality assurance (QA) requirements. If a single QA requirement was not met, the batch had to be abandoned. In settings (1) and (2) the two-stage sampling described above was used. Casual conversations with colleagues suggest that in other contexts this two-stage sampling is also not unusual.

Indeed, there is an almost irresistible temptation to try to get more measurements, when the first set of measurements suggests that a severe cost could have been avoided if only more measurements had initially been made. In this note we examine the price of yielding to the temptation, and provide a mathematically correct way to do it by adjusting the value of $\alpha$.

\subsection{Related Literature}

Hewett and Spurrier (1983) -ve a review of two-stage methods. For the situation described in Section 1.1, they base their decision to stop or continue only on the UCL from the first stage, not on the sample mean. On the second stage, they do not necessarily use the same nominal $\alpha$ as on the first stage, although the two nominal 
$\alpha$ 's are chosen to give the procedure the desired overall properties. This freedom to choose two sample sizes and two $\alpha$ 's allows them, in principle, to adjust the parameters of the procedure to produce a power curve that is determined at three points, although the computations are not easy.

Jennison and Turnbull (1990) give an easily readable review of multi-stage methods as applied in medical trials. In that context, there can be both economic and ethical reasons for wanting to terminate experiments early if possible. One of the articles cited, by Pocock (1977), uses the easy formulation of $N$ groups each containing $n$ measurements; the experiment can be terminated after any of the $N$ sets of measurements. Pocock uses symmetrical two-sided tests, which differ from those of this report, but his computational method could probably be modified for the setting here. Another approach in the medical literature, known as "stochastic curtailment", considers the conditional power, that is, the power of the final statistical procedure conditional on the data already obtained. The test is terminated if the conditional power is close to 0.0 or 1.0 , that is, if the final conclusion will probably agree with the preliminary conclusion. This is related in spirit to the method described below in Section 4.2 , in which the sample size needed on the second stage is estimated from the first-stage data.

In industrial acceptance sampling, two-stage and sequential ideas seem to be used when the data consist of counts of defective cases but not when the data consist of measurements of continuous variables. For example, two-stage sampling (called "double sampling") is mentioned in the chapter on acceptance sampling in Juran and Gryna (1988), when the data consist of counts of defective items, and Military Standards are cited and explained. However, when continuous measurements are taken a second sample is considered only by re-evaluating the sample as a count of defective items. Similarly, Duncan (1965) describes double sampling in situations when the data consist of counts of defective items, but he does not mention double sampling when the data consist of continuous measurements.

The situation in environmental contexts seems to be similar. Wald's sequential probability ratio test is cited by an EPA (1989) report, when the data consist of counts of acceptable and unacceptable cases. However the EPA example cited in Section 1.1 
above shows no awareness of sequential ideas when the data consist of measurements of continuous variables, and we have not found cases where two-stage or sequential sampling is correctly used with such data in environmental settings.

We have not compared in detail the procedures of this note to the many others that can be found in the literature. The purpose of this note is not to find an optimal two-stage procedure, or even to advocate use of a two-stage procedure over a one-stage procedure, but to alert applied workers to the consequences of using the apparently widespread two-stage procedure described in Section 1.1.

\section{TERMINOLOGY}

Assume that all the observations $X_{i}$ are independent and normally distributed with mean $\mu$ and variance $\sigma^{2}$. The (regulatory or QA) requirement is met if the $100(1-$ $\alpha) \%$ UCL on $\mu$ is less than some limit $L$.

The two-stage procedure is stated formally as follows, for $\alpha<0.5$. Let $n_{1}$ measurements be taken, resulting in sample mean and sample standard deviation denoted by $\bar{X}_{1}$ and $s_{1}$. Define the following events $A 1, R 1$, and $C 1$. The names stand for "accept," "reject," and "continue," and the number 1 denotes the first stage of sampling:

$A 1: \quad \bar{X}_{1}+t_{1-\alpha}\left(n_{1}-1\right) s_{1} / \sqrt{n_{1}}<L$

R1: $\quad \bar{X}_{1} \geq L$

$C 1: \quad \bar{X}_{1}+t_{1-\alpha}\left(n_{1}-1\right) s_{1} / \sqrt{n_{1}} \geq L \quad$ and $\quad \bar{X}_{1}<L$

Note that for any sample, exactly one of the three events occurs. If $A 1$ occurs, declare that the requirement is met, i.e., accept the population. If instead $R 1$ occurs, declare that the requirement is not met, i.e., reject the population. If the third possibility $C 1$ occurs, take a second sample of size $n_{2}$. Let $n=n_{1}+n_{2}$, and let $\bar{X}$ and $s$ be the sample mean asu sample standard deviation based on all $n$ measurements. Then derine

A2: $\quad \bar{X}+t \cdot{ }_{a}(n-1) s / \sqrt{n}<L$

R2: $\quad \bar{X}+t_{1-\alpha}(n-1) s / \sqrt{n} \geq L$ 
If $A 2$ occurs, accept the population. Otherwise (that is, if $R 2$ occurs), reject it.

It has not yet been stated how $n_{2}$ is chosen. This note considers two possibilities: either $n_{2}$ is fixed in advanced ("prespecified"), or $n_{2}$ depends on the measurements taken in the first stage of sampling. This issue is a source of possible confusion, so we elaborate here. Whether to take a second sample at all certainly depends on the results of the first stage of sampling. That is not being discussed here. The question here is how many measurements to take in the second sample, if a second sample is to be taken. This sample size $n_{2}$ may be set in advance, or it may be calculated from the first sample. For the calculations in Section 4, we consider both possibilities. Possible prespecified values of $n_{2}$ are $n_{1} / 2, n_{1}, 2 n_{1}$, etc. If instead $n_{2}$ is calculated from the first sample, the type of formula used is

$n=\left[t_{1-\alpha}\left(n_{1}-1\right) s_{1} /\left(L-\bar{X}_{1}\right)\right]^{2}+$ a few more to be safe.

The first term on the right is rounded to the nearest integer, and the "few more" term is $n_{1} / 2$ or something similar. Then $n_{2}$ is found as $n-n_{1}$.

This formulation in terms of a confidence limit and acceptance or rejection of a population is put in a standard hypothesis-testing framework by defining

$H_{0}: \mu \geq L$

$H_{1}: \mu<L$.

Acceptance of the null hypothesis corresponds to rejection of the population. The probability of a Type I error for this procedure is the probability of accepting the population when $\mu=L$. That is

$P($ Type I error $)=P(A 1)+\mathrm{P}(C 1) P(A 2 \mid C 1)$

where $\mu$ is assumed to equal $L$. The purpose of this note is to compare $P$ (Type I error) to the nominal value $\alpha$. We will always use $\alpha$ as a parameter, a nominal value used to obtain a $t$-value; we will always write out the true probability as $P$ (Type I error). 
The rule for what to do on the first stage can be restated in terms of a $p$-value, defined as

$p=P\left(\bar{X}_{1}+t_{1-\alpha}\left(n_{1}-1\right) s_{1} / \sqrt{n_{1}}<L \mid \mu=L\right)$.

Then the rule is: accept the population (reject $H_{0}$ ) if $p<\alpha$, reject the population (accept $H_{0}$ ) if $p>1 / 2$, and continue to the second stage otherwise. This could be generalized by replacing $1 / 2$ by some value selected between $\alpha$ and 1 , but we do not pursue this here.

\section{APPROXIMATIONS AND BOUNDS}

Assume here that $\mu=L$. Assume also that $\alpha<0.5$, because the two-stage procedure based on $\bar{X}_{1}$ would only be used if $\alpha<0.5$. Note first that

$P(A 1)=\alpha$

and

$$
\begin{aligned}
P(C 1)= & P\left[\bar{X}_{1}+t_{1-\alpha}\left(n_{1}-1\right) s / \sqrt{n_{1}}>L \text { and } \bar{X}_{1}<L\right] \\
= & P\left[\bar{X}_{1}+t_{1-\alpha}\left(n_{1}-1\right) s / \sqrt{n_{1}}>L\right]- \\
& P\left[\bar{X}_{1}+t_{1-\alpha}\left(n_{1}-1\right) s / \sqrt{n_{1}}>L \text { and } \bar{X}_{1} \geq L\right] \\
= & P\left[\bar{X}_{1}+t_{1-\alpha}\left(n_{1}-1\right) s / \sqrt{n_{1}}>L\right]-P\left[\bar{X}_{1} \geq L\right] \\
= & (1-\alpha)-0.5 \\
= & 0.5-\alpha
\end{aligned}
$$

Therefore, Equation (1) yields

$P($ Type I error $)=\alpha+(0.5-\alpha) P(A 2 \mid C 1)$

and

$\alpha \leq P$ (Type I error $) \leq \alpha+(0.5-\alpha)=0.5$ 
The above inequality is valid whether $n_{2}$ is prespecified or random. When $n_{2}$ is prespecified, approximations can be found if $n_{2} \gg n_{1}$ or if $n_{2} \ll n_{1}$. In the first case, the second sample overwhelms the first, and $P(A 2 \mid C 1) \doteq \alpha$. Therefore

$P($ Type I error $) \doteq \alpha+(0.5-\alpha) \alpha=1.5 \alpha-\alpha^{2}$ for prespecified $n_{2} \gg n_{1}$.

In the case where $n_{2} \ll n_{1}$, the second sanple has virtually no effect; if the first sample does not cause the population to be accepted, neither will the pooled sample. Therefore, $P(A 2 \mid C 1) \doteq 0$, and

$P($ Type I error $) \doteq \alpha$ for prespecified $n_{2} \ll n_{1}$.

It might be natural to expect $P$ (Type I error) to vary monotonically as $n_{2}$ increases from 1 to infinity. The results of Section 4.1 show that this is not the case. Therefore the approximation (2) $\therefore$... $l y$ y a limiting approximation, not an upper bound.

\section{NUMERICAL RESULTS}

For selected two-stage sampling schemes, tables here give the nominal $\alpha$ to use to obtain the desired true Type 1 error. Power curves for these schemes are also shown. Section 4.1 deals with prespecified $n_{2}$ and Section 4.2 deals with random $n_{2}$.

\subsection{Prespecified $n_{2}$}

Consider first a two-stage sampling scheme in which $n_{2}$ is specified in advance. The acceptance rule uses some nominal $\alpha$, and the true $P$ (Type I error) can be calculated by numerical integration, as explained in Section A.1 of Appendix A.

An example of the kind of result obtained for $\alpha=0.10$ is shown in Figure 1. The horizontal axis shows $n_{2}$ as a multiple of $n_{1}$, so that the one graph can show results for 


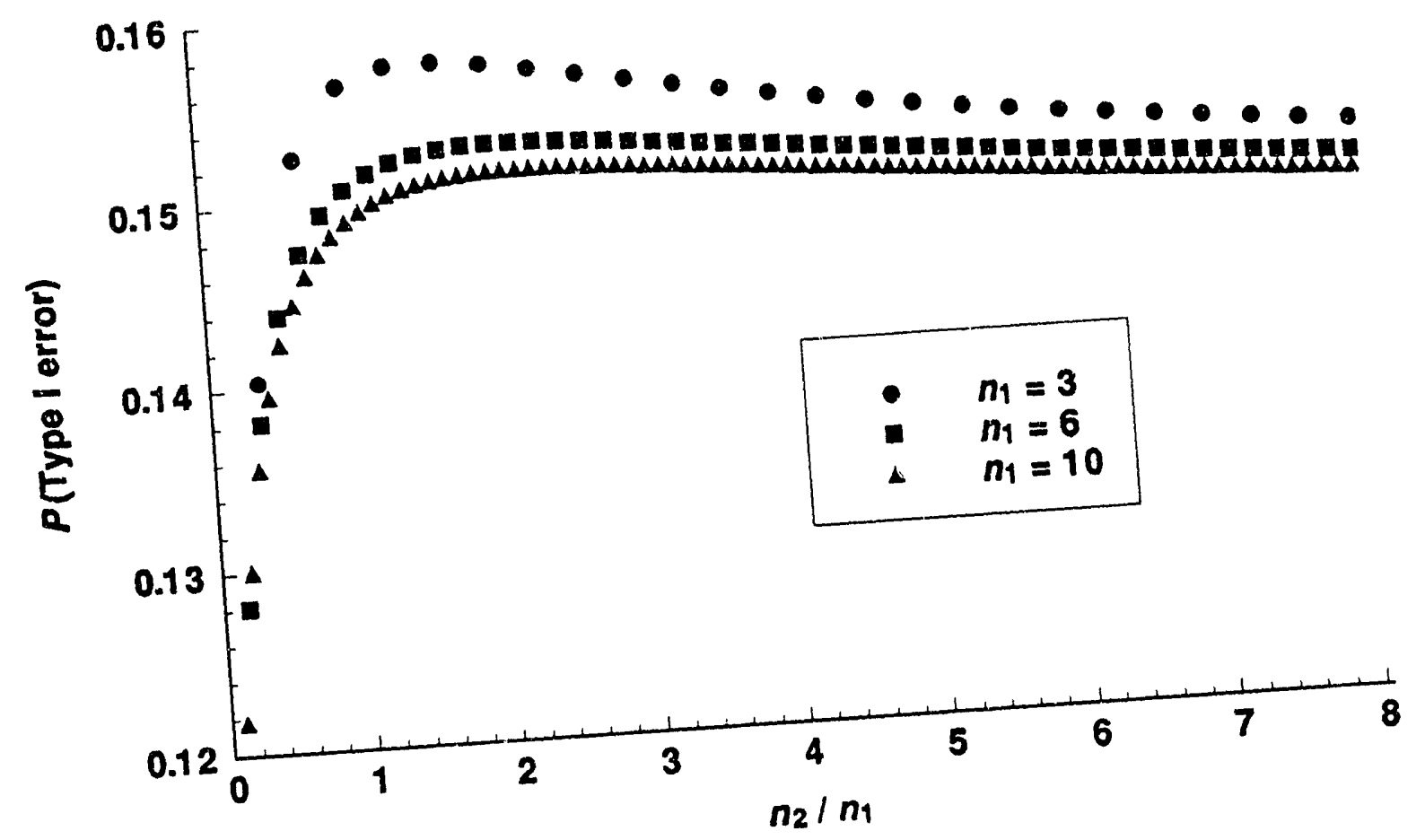

Figure 1. Effect of $n_{2}$ on $P$ (Type I error) when $\alpha=0.10$.

several values of $n_{1}$. Note that $P$ (Type $I$ error) rises to a maximum in the vicinity of $n_{2}=2 n_{1}$, and then falls very slowly. The limiting value of Equation (2) is 0.14 ; the figure shows that $n_{2}$ must be much larger than $8 n_{1}$ before $P$ (Type I error) is close to this limiting value. We see that crude empirical bounds are given by

$\alpha<P$ (Type I error) $<2 \alpha$.

The corresponding figures for $\alpha=0.05$ and $\alpha=0.01$ are qualitatively similar to Figure 1: the curves all lie between $\alpha$ and $2 \alpha$, rising to a maximum near $n_{2}=2 n_{1}$ and then falling slowly.

Once this calculation was programmed, a numerical search was used to find the value of $\alpha$ that led to the desired P(Type I error). These values are given in Table ${ }^{1}$ for various $n_{2}$. 
Table 1. Nominal $\alpha$ and multipliers ${ }^{a}$ yielding desired $P$ (Type I error), when sampling with prespecified $n_{2}$.

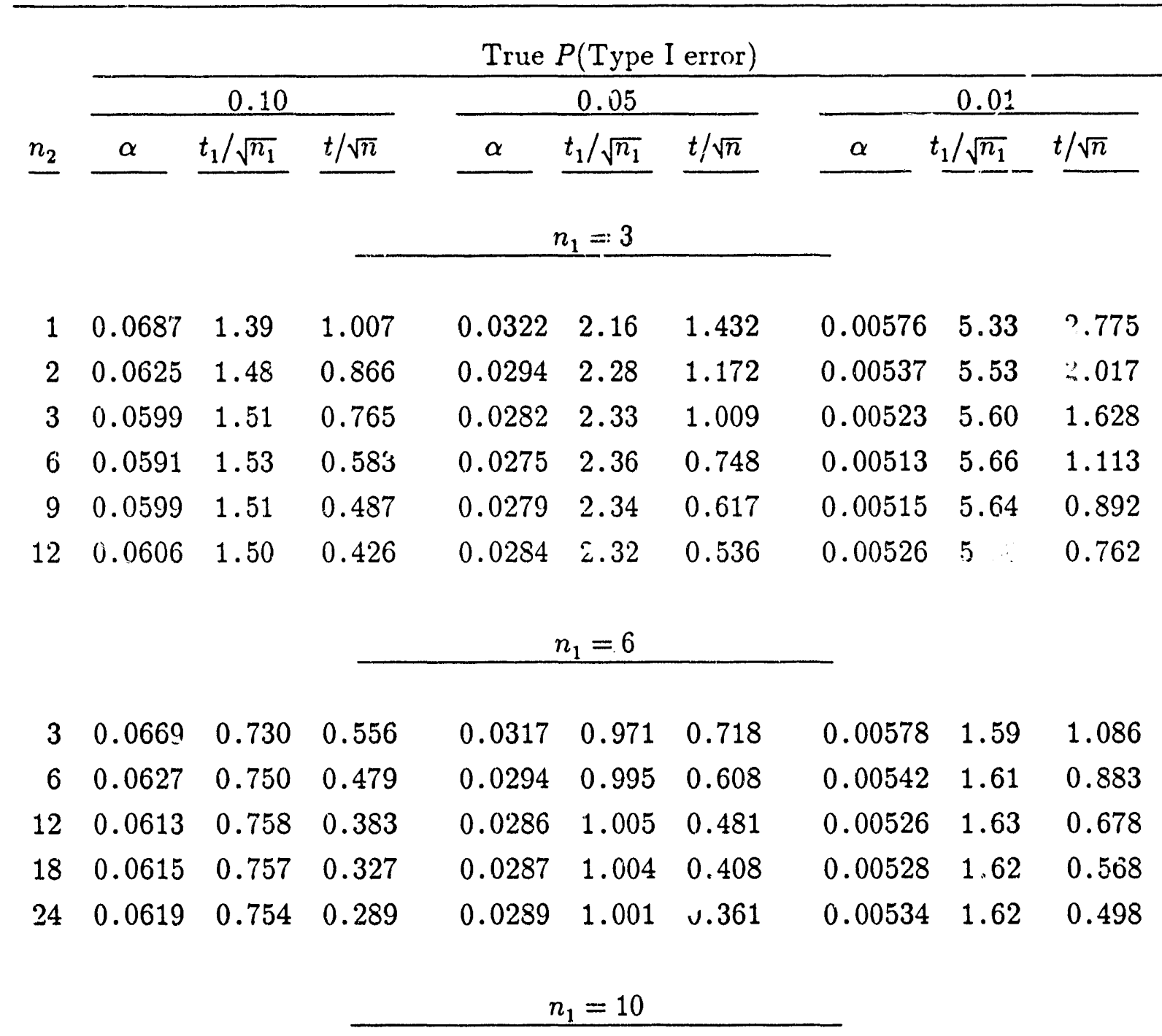

$\begin{array}{rlllllllll}5 & 0.0678 & 0.518 & 0.409 & 0.0322 & 0.666 & 0.518 & 0.00591 & 0.995 & 0.747 \\ 10 & 0.0638 & 0.531 & 0.356 & 0.0299 & 0.680 & 0.447 & 0.00552 & 1.008 & 0.630 \\ 20 & 0.0622 & 0.536 & 0.289 & 0.0290 & 0.687 & 0.360 & 0.00533 & 1.015 & 0.498 \\ 30 & 0.0622 & 0.536 & 0.248 & 0.0290 & 0.687 & 0.309 & 0.00534 & 1.015 & 0.424 \\ 40 & 0.0625 & 0.535 & 0.221 & 0.0292 & 0.686 & 0.274 & 0.00539 & 1.013 & 0.375\end{array}$

a. The multipliers are $t_{1-\alpha}\left(n_{1}-1\right) / \sqrt{n_{1}}$ on the first stage, and $t_{1-\alpha}(n-1) / \sqrt{n}$ on the second stage, with $n=n_{1}+n_{2}$. 
For example, if we decide to let $n_{1}=10$ and $n_{2}=40$, and if $P$ (Type I error) is to equal 0.10 , Table 1 shows that the nominal value of $\alpha$ to use is 0.0625 . Figure 2 shows the expected sample size for this procedure, and Figure 3 shows the power curve. Here all measurements have been shifted so that the regulatory or QA limit is 0.0 , and the standard deviation of the masurements is 1.0. Alternatively, $\mu$ measures the distance from the regulatory limit $L$ in $\sigma$-unirs. The power curve has height 0.10 at $\mu=0.0$, as it should. Nete that the expected sample size depends on $\mu$ : if $\mu$ is far from the regulatory limit of 0 , the decision will be clear after the first sample, and the expected sample size will be approximately $n_{1}$; if instead $\mu$ is a little less than the regulatory limit, the second sample will often be necessary, and the expected sample sixe will be $n_{1}+P(\mathrm{C} 1) n_{2}>n_{1}$.

The value 27 is shown in Figure 2. This is approximately the expected sample size when $\mu$ equals the regulatory limit of 0 . Figure 3 shows the power curve for both the two-stage procedure and for the one-stage procedure with $n=27$. Note that the two-stage procedure has better power for $-0.61<\mu<0.0$, and that this range is very slightly larger than the region where the two-stage procedure has a larger expected sample size, $-0.55<\mu<0.01$. That is, there are two factors influencing which procedure has the larger power: roughly, the procedure with the larger expected sample size has the larger power; however, the sequential nature of the two-stage proceduce gives it a slight advantage, which is evident when the two expected sample sizes are approximately equal.

\subsection{Random $\mathrm{n}_{2}$}

Consider a two-stage sampling scheme in which $n_{2}$ is calculated from the first sample. The acceptance rule uses some nominal $\alpha$, and the true $P$ (Type I error) can be estimated from Monte Carlo simulation. This was done for selected sampling schemes, and a 1 imerical search procedure was then used to find the value $u ; \alpha$ that yielded desired values of $P$ (Type I error) such as 0.10 or 0.05 . The values are shown in Table 2 . For convenience, the values of $t_{1-\alpha}\left(n_{1}-1\right)$ are also shown. The $t$-value for the second stage $t_{1-\alpha}(n-1)$ cannot be printed, because the random value of $n$ depends on the first 


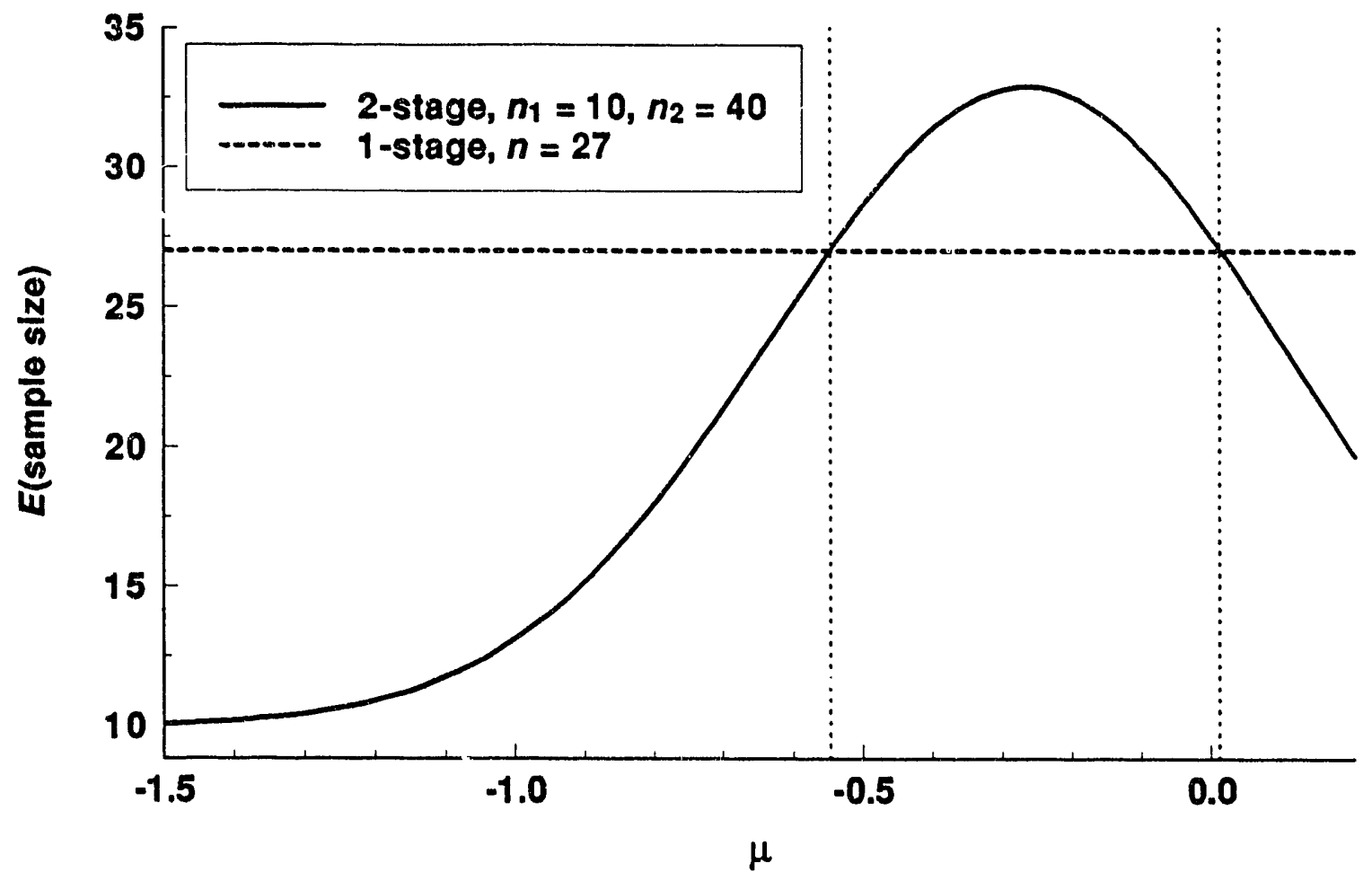

Figure 2. Expected sample size of two-stage pr ,cedure with prespecified $n_{2}$ and of onestage procedure.

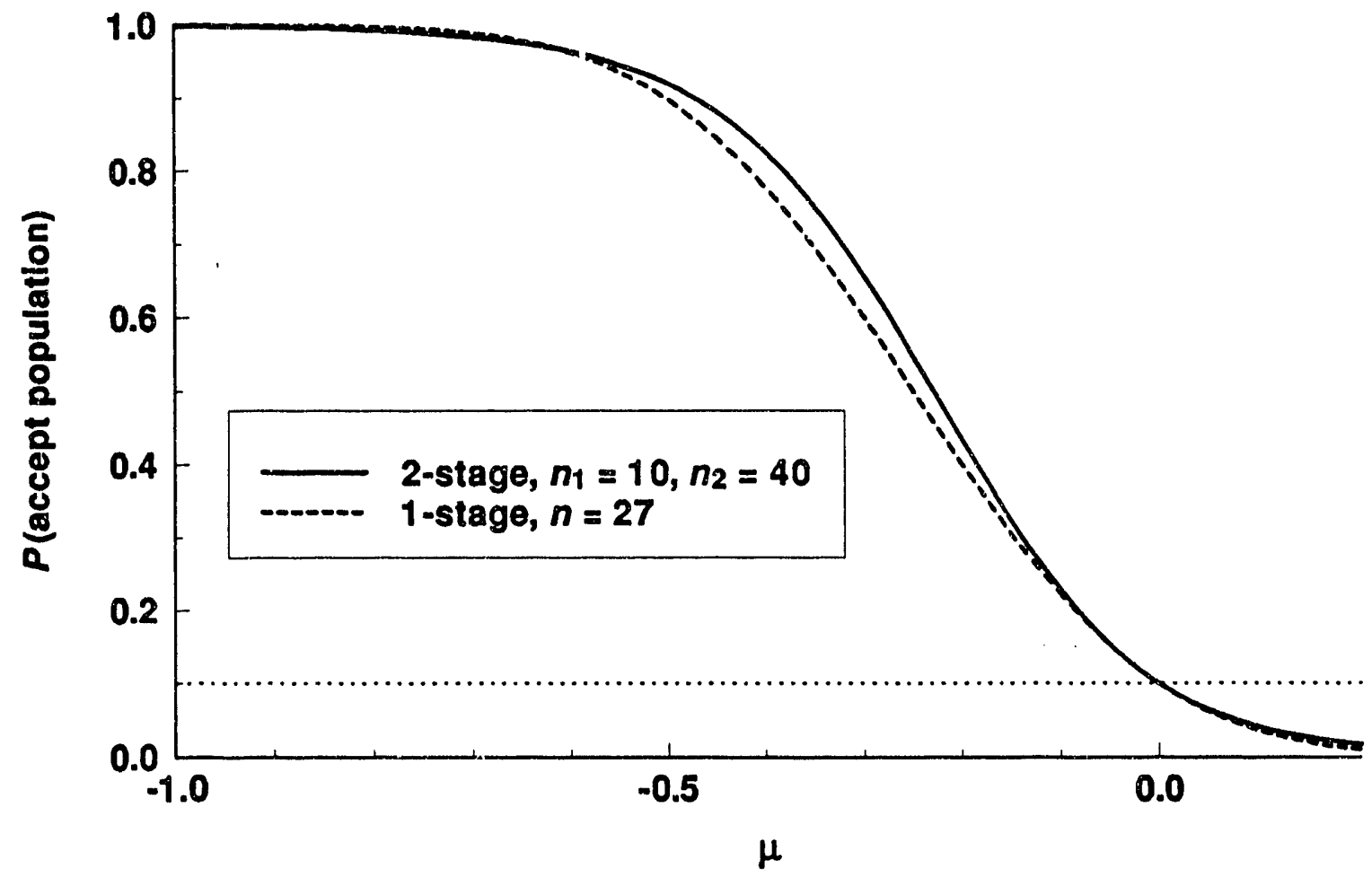

Figure 3. Power curve of two-stage procedure with prespecified $n_{2}$ and of one-stage procedure. 
Table 2. Nominal $\alpha$ and $t_{1-\alpha}\left(n_{1}-1\right)^{a}$ yielding desired $P$ (Type I error), when sampling with random $n_{2}{ }^{b}$

\begin{tabular}{|c|c|c|c|}
\hline \multirow[b]{2}{*}{$n_{1}$} & \multicolumn{3}{|c|}{ True $P$ (Type I error) } \\
\hline & 0.10 & 0.05 & 0.01 \\
\hline & & $w=1$ & \\
\hline 3 & $0.0841,2.12$ & $0.0419,3.23$ & $0.00882,7.43$ \\
\hline 6 & $0.0867,1.59$ & $0.0421,2.15$ & $0.00835,3.53$ \\
\hline \multirow[t]{2}{*}{10} & $0.0870,1.48$ & $0.0420,1.94$ & $0.00812,2.95$ \\
\hline & & $w=2$ & \\
\hline 3 & $0.0773,2.24$ & $0.0385,3.39$ & $0.00837,7.63$ \\
\hline 6 & $0.0778,1.67$ & $0.0371,2.25$ & $0.00728,3.66$ \\
\hline \multirow[t]{2}{*}{10} & $0.0779,1.55$ & $0.0369,2.02$ & $0.00690,3.05$ \\
\hline & & $w=4$ & \\
\hline 3 & $0.0714,2.35$ & $0.0354,3.56$ & $0.00789,7.87$ \\
\hline 6 & $0.0711,1.74$ & $0.0335,2.33$ & $0.00645,3.78$ \\
\hline \multirow[t]{2}{*}{10} & $0.0713,1.61$ & $0.0332,2.09$ & $0.00608,3.13$ \\
\hline & & $w=8$ & \\
\hline 3 & $0.0666,2.46$ & $0.0329,3.70$ & $0.00745,8.10$ \\
\hline 6 & $0.0665,1.79$ & $0.0309,2.40$ & $0.00586,3.87$ \\
\hline 10 & $0.0668,1.65$ & $0.0308,2.14$ & $0.00554,3.19$ \\
\hline
\end{tabular}

a. The tabulated values for $\alpha$ were obtained by Monte Carlo sirnulation. For each entry, with $95 \%$ confidence the error in $\alpha$ is at most \pm 2 in the final digit, and the error in $t_{1-\alpha}\left(n_{1}-1\right)$ is at most \pm 1 in the final digit.

b. The second sample size $n_{2}$ is based on Equation (3), constrained by $n_{2} \leq w n_{1}$. 
sample; once $n$ has been determined, the $t$-value can be found using any of a number of commercial statistics computer packages.

For the tabulated values, the second sample size was calculated from the first sample by the formula

$n=\left[t_{1-\alpha}\left(n_{1}-1\right) s_{1} /\left(L-\bar{x}_{1}\right)\right]^{2}+$ a few more.

The squared term on the right was rounded to the nearest integer. For the "few more," the values $n_{1}$ and $n_{1} / 2$ were initially considered, but preliminary simulations showed little difference in the power curves for the two cases. Therefore only the less restrictive value of $n_{1} / 2$ was used in Table 2, leading to

$n=\left[t_{1-\alpha}\left(n_{1}-1\right) s_{1} /\left(L-\bar{x}_{1}\right)\right]^{2}+n_{1} / 2$.

When $n_{1}=3, n_{1} / 2$ was truncated to 1 . Equation (3) defines $n$, and therefore defines $n_{2}$ as $n-n_{1}$.

In practice there is a maximum $n_{2}$, above which further sampling is not worth the trouble. This maximum is expressed as $w n_{1}$, and the values $w=1,2,4$, and 8 were considered. If Equation (3) resulted in $n_{2}$ greater than this allowed $w n_{1}$, the population was rejected without further sampling.

A good case for illustration is when $P$ (Type I error) $=0.10$ and $n_{1}=10$. Consider the two extreme cases of $w=1$ (with $\alpha=0.0870$ ) and $w=8$ (with $\alpha=0.0668$ ). These two procedures result in expected sample sizes shown in Figure 4 and power curves shown in Figure 5. As with Figures 2 and 3, all measurements have been shifted here so that the regulatory or QA limit is 0.0 , and the standard deviation of the measurements is 1.0. The power curves have height 0.10 at $\mu=0.0$, as they should. As would be expected, using $w=8$ yields a larger average sample size than $w=1$, and herefore a more discriminating test - a higher power curve to the left of zero and a lower one to the right. 


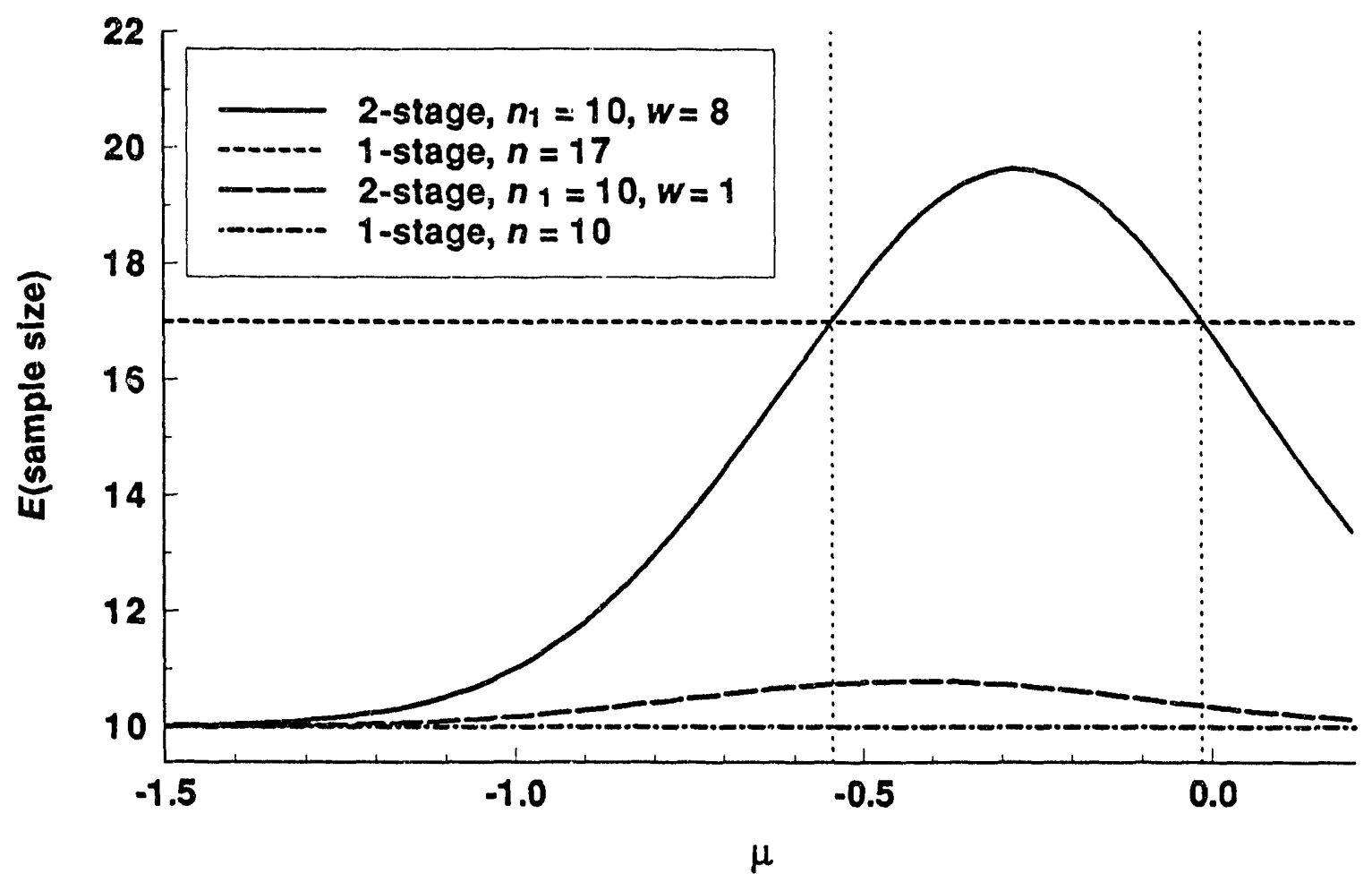

Figure 4. Expected sample sizes of two-stage procedures with random $n_{2}$ and of onestage procedures.

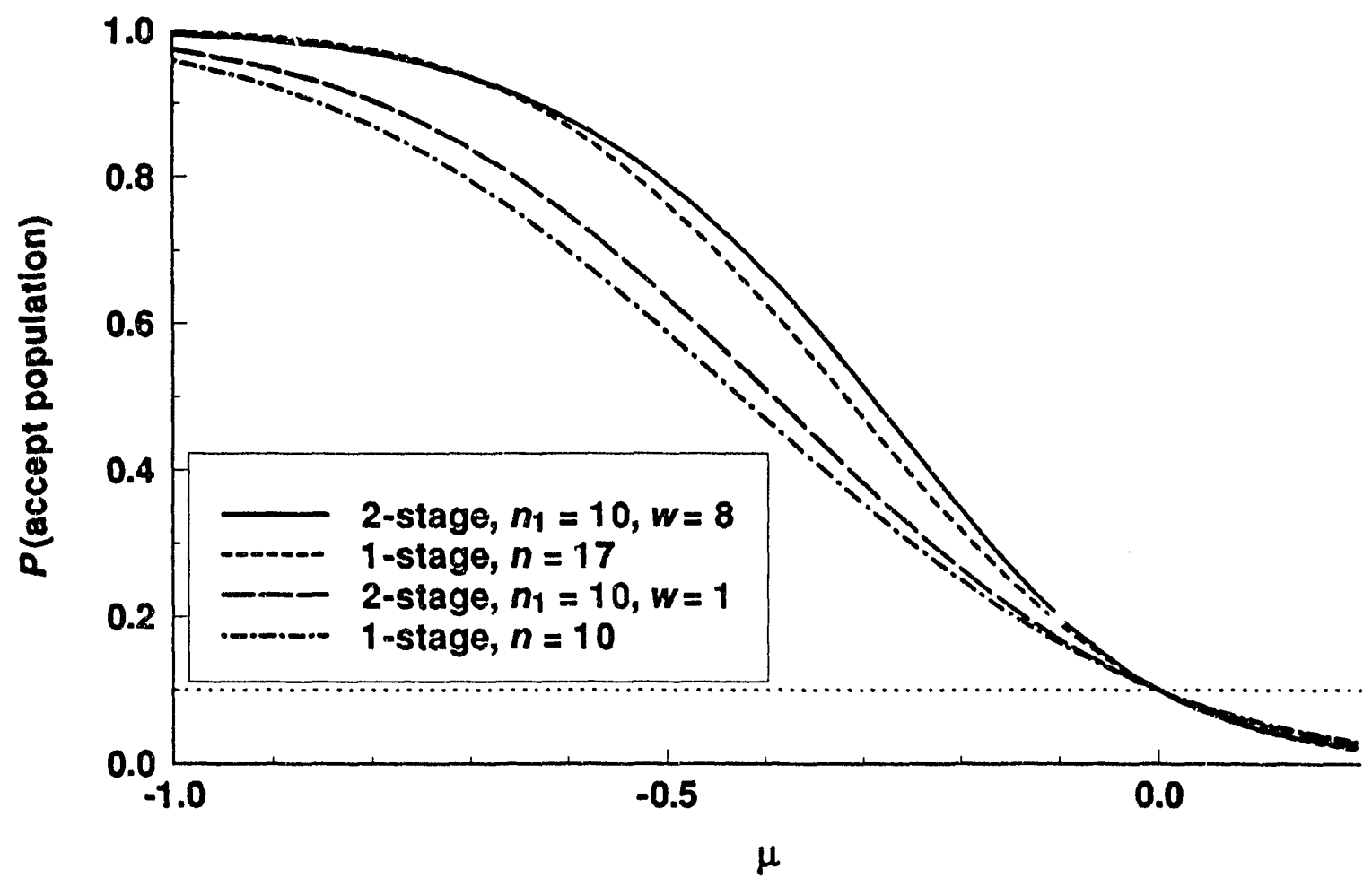

Figure 5. Power curves of two-stage procedures with random $n_{2}$ and of one-stage procedures. 
For comparison, two tests with fixed sample size are also considered. The procedure with $n=10$ (i.e. always stop after the first sample) has properties not too different than the two-stage procedure with $w=1$. The two-stage procedure has an average sample size slightly larger than 10 , and a power curve slightly higher than that of the fixed-size procedure. The case with $n=17$ was selected because the average sample size is approximately 17 for $w=8$ and $\mu=0$. The two-stage procedure with $w=8$ has a larger power than the one-stage procedure in approximately that region of the $\mu$-axis where the two-stage sample size is larger than 17 .

In Table 2, it is surprising that the values of $\alpha$ are not all monotone in $n_{1}$, for fixed $w$ and $P$ (Type I error). The values are believed to be correct, however: the program for simulating $P$ (Type I crror) is quite direct, and has been carefully reviewed by both authors. It is explained in Section A.2 of the Appendix.

\section{CORRECT TWO-STAGE SAMPLING}

Decide in advance whether two-stage sampling is to be used or not. It is incorrect to use two-stage sampling only for data sets where the two-stage procedure appears advantageous. Therefore, decide for all the data sets in the project whether one-stage or two-stage sampling is to be used, and apply this procedure consistently.

If two-stage sampling is used, decide whother the second sample size is prespecified or calculated from the first sample. If calculated from the first sample, decide how large a second sample would be considered, as a multiple of the first sample. Based on Table 1 or Table 2, use the nominal $\alpha$ yielding the desired true $P$ (Type I error).

To illustrate the method, reconsider the example from SW-846 described in Section 1. Suppose that two-stage sampling is to be used, with prespecified sample sizes $n_{1}=6$ and $n_{2}=3$. As shown in Section $4.1, \alpha=0.0669$ yields $P$ (Type I error) $=0.10$. Therefore 


$$
\begin{aligned}
\mathrm{UCL} & =\bar{X}_{1}+t_{0.9331}\left(n_{1}-1\right) s_{1} / \sqrt{n_{1}} \\
& =94.67+0.730 \times 9.52 \\
& =101.62 \mathrm{ppm} .
\end{aligned}
$$

This is larger than the regulatory limit of 100 , although the sample mean is smaller than the limit. Therefore a second sample should be taken.

Based on the full sample of nine measurements, we have

$$
\begin{aligned}
\mathrm{UCL} & =\bar{X}+t_{0.9331}(n-1) s / \sqrt{n} \\
& =93.56+0.556 \times 7.75 \\
& =97.87 \mathrm{ppm} .
\end{aligned}
$$

This is less than the regulatory limit of 100 , so we conclude with $90 \%$ confidence that the population mean is less than the regulatory limit of 100 ppm, i.e., the regulation is satisfied. The use of nominal $\alpha=0.0669$ yielded a true $P$ (Type I error) $=0.10$ when two-stage sampling was used.

This example could also be worked out with $n_{2}$ calculated based on the first sample. In this case, however, the total sample size is calculated by Equation (3), constrained by $n_{2} \leq w n_{1}$. The tone of SW-846 suggests that the second sample size should not be large. Therefore we use $w=1$, and Table 2 says to use a nominal $\alpha$ of 0.0867 . This results in

$$
\begin{aligned}
n & =\left[t_{1-\alpha}(5) 9.52 / 5.33\right]^{2}+3 \\
& =[1.59 \times 9.52 / 5.33]^{2}+3 \\
& \doteq 11
\end{aligned}
$$

Therefore, Table 2 requires a second sample larger than taken in the published example, so the illustrative calculations cannot be completed. 


\section{REFERENCES}

Duncan, Acheson J. (1965), Quality Control and Industrial Statistics, Third Edition, Homewood, IL: Richard D. Irwin, Inc.

EPA (1986), Test Methods for Evaluating Solid Waste, Third Edition, Volume II: Field Manual, Physical/Chemical Methods, SW-846, U.S. Environmental Protection Agency.

EPA (1989), Methods for Evaluating the Attainment of Cleanup Standards, Volume I: Soils and Solid Media, EPA 230/02-89-042, Chapter 8.

Jennison, Christopher, and Bruce W. Turnbull (1990), "Statistical Approaches to Interim Monitoring of Medical Trials: A Review and Commentary," Statistical Science, 5, 3, pp. 299-317.

Juran, J. M., and Frank M. Gryna (1988), Juran's Quality Control Handbook, Fourth Edition, New York: McGraw-Hill, pp. 25.21-25.68.

Hewett, John E., and John D. Spurrier (1983), "A Survey of Two Stage Tests of Hypotheses: Theory and Application," Communications in Statistics - Theory and Methods, 12, 20, pp. 2307-2425.

Pocock, Stuart J. (1977), "Group Sequential Methods in the Design and Analysis of Clinical Trials," Biometrika, 64, 2, pp. 191-199. 


\section{APPENDIX: DETAILS OF COMPUTATIONS}

\section{A.1 Integration with Prespecified $n_{2}$}

By changing the units of $X$, one can let the regulatory or QA limit $L$ equal 0 and let $\sigma^{2}=1$ with no loss of generality. This is done here without further comment.

\section{A.1.1 Interation Limits for $P$ (Type I error)}

Assume here that $n_{2}$ is prespecified, fixed in advance. The probability of accepting the population on the first stage, $P(A 1)$, is $\alpha$. The probability of continuing and then accepting the population on the second stage, $P(C 1$ and $A 2)$, can be evaluated by integration of the joint density of the observations $X_{i}$. Because the measurements are independent and identically distributed normal $(0,1)$, the joint density can be reduced to one in terms of the mutually independent random variables $\bar{X}_{1},\left(n_{1}-1\right) s_{1}{ }^{2}$, $\bar{X}_{2}$, and $\left(n_{2}-1\right) s_{2}{ }^{2}$, where $\bar{X}_{i}$ and $s_{i}{ }^{2}$ are the sample mean and variance from the $i$ th sample, with $i=1$ or 2 . Each $\bar{X}_{i}$ has a normal distribution with mean 0 and variance $1 / n_{i} ;\left(n_{i}-1\right) s_{i}{ }^{2}$ has a $\chi^{2}$ distribution with $\left(n_{i}-1\right)$ degrees of freedom. The joint density of the four random variables is the product of individual densities.

The desired probability can be evaluated as an iterated integral, with the region of integration developed here. Let $t_{m}$ denote $t_{1-\alpha}(m-1)$, the $100(1-\alpha)$ percentile of Student's $t$ distribution with $m-1$ degrees of freedom, for $m=n_{1}$ or $m=n \equiv n_{1}+n_{2}$. Define the following events:

$$
\begin{array}{ll}
E_{1}: & \bar{X}_{1}<0 \\
E_{2}: & \bar{X}_{1}+t_{n_{1}} s_{1} / \sqrt{n_{1}}>0 \\
E_{3}: & \bar{X}<0 \\
E_{4}: & \bar{X}+t_{n} s / \sqrt{n}<0
\end{array}
$$

Note that $C 1 \equiv E_{1} \cap E_{2}$ and that $C 1 \cap A 2 \equiv E_{1} \cap E_{2} \cap E_{3} \cap E_{4}$. Event $E_{1}$ defines the bounds of integration for $\bar{x}_{1}$ : 
$\bar{x}_{1}<0$.

Given $\bar{x}_{1}<0$, the limits of integration for $\left(n_{1}-1\right) s_{1}{ }^{2}$ can be derived from $E_{2}$ as follows.

$$
\begin{array}{rlr}
\bar{x}_{1} & +t_{n_{1}} s_{1} / \sqrt{n_{1}}>0 & \\
& \Leftrightarrow s_{1}>-\bar{x}_{1} \sqrt{n_{1}} / t_{n_{1}} & \\
& \Leftrightarrow s_{1}{ }^{2}>n_{1}\left(\bar{x}_{1} / t_{n_{1}}\right)^{2} & \text { because } \bar{x}_{1}<0 \\
& \Leftrightarrow\left(n_{1}-1\right) s_{1}{ }^{2}>n_{1}\left(n_{1}-1\right)\left(\bar{x}_{1} / t_{n_{1}}\right)^{2} . &
\end{array}
$$

Sir.tilarly given $\bar{x}_{1}$, the limits of integration for $\bar{x}_{2}$ can be derived from $E_{3}$ as follows

$$
\begin{aligned}
\bar{x}<0 & \\
& \Leftrightarrow \frac{n_{1} \bar{x}_{1}+n_{2} \bar{x}_{2}}{n}<0 \\
& \Leftrightarrow \bar{x}_{2}<-n_{1} \bar{x}_{1} / n_{2} .
\end{aligned}
$$

Finally the region for $\left(n_{2}-1\right) s_{2}{ }^{2}$ can be derived as follows, although the algebra is somewhat more complicated. Given $\bar{x}<0$, event $E_{4}$ implies that

$$
(n-1) s^{2}<n(n-1)\left(\bar{x} / t_{n}\right)^{2} .
$$

This is the analogue of inequality (A-2). It must be rewritten in terms of $\left(n_{2}-1\right) s_{2}{ }^{2}$. Observe that

$$
\begin{aligned}
(n-1) s^{2} & =\sum_{i=1}^{n_{1}}\left(x_{i}-\bar{x}\right)^{2}+\sum_{i=n_{1}+1}^{n}\left(x_{i}-\bar{x}\right)^{2} \\
& =\left(n_{1}-1\right) s_{1}{ }^{2}+n_{1}\left(\bar{x}_{1}-\bar{x}\right)^{2}+\left(n_{2}-1\right) s_{2}{ }^{2}+n_{2}\left(\bar{x}_{2}-\bar{x}\right)^{2} \\
& =\left(n_{1}-1\right) s_{1}{ }^{2}+\left(n_{2}-1\right) s_{2}{ }^{2}+\left(n_{1} n_{2} / n\right)\left(\bar{x}_{1}-\bar{x}_{2}\right)^{2}
\end{aligned}
$$

Substitution of this into (A-4) yields, after rearrangement,

$$
\left(n_{2}-1\right) s_{2}{ }^{2}<n(n-1)\left(\bar{x} / t_{n}\right)^{2}-\left(n_{1}-1\right) s_{1}{ }^{2}-\left(n_{1} n_{2} / n\right)\left(\bar{x}_{1}-\bar{x}_{2}\right)^{2} .
$$


In summary, the limits of integration for evaluating $P(C 1$ and $A 2)$, from the outermost integral to the innermost, are given by inequalities (A-1), (A-2), (A-3), and (A-6). The corresponding variables of integration are $\bar{x}_{1},\left(n_{1}-1\right) s_{1}{ }^{2}, \bar{x}_{2}$, and $\left(n_{2}-1\right) s_{2}{ }^{2}$. The integrand is the product of the four densities, normal $\left(0,1 / n_{1}\right)$, $\chi^{2}\left(n_{1}-1\right)$, normal $\left(0,1 / n_{2}\right)$, and $\chi^{2}\left(n_{2}-1\right)$.

If the computer software has a function to evaluate the $\chi^{2}$ cumulative distribution function, the innermost integral should be evaluated by this function instead of by direct numerical integration. This reduces the quadruple integral to a triple integral. Even evaluating the triple integral was challenging, because the regions of integration had to be partitioned so that the computational program could concentrate its efforts in regions where the greatest accuracy was necessary. Without this partitioning, the integrations could take much too long to be feasible.

\section{A.1.2 Discussion}

Let $\alpha_{\text {want }}$ be the desired $P$ (Type I error). Table 1 was calculated by first setting $\alpha=\alpha_{\text {want }}$ and $\alpha=\alpha_{\text {want }} / 2$, and computing the resulting values of $P$ (Type I error) by the integration method of Section A.1.1. The two calculated probabilities bracketed $\alpha_{\text {want }}$. Then the method of false position was used to find successively better values of $\alpha$, until an $\alpha$ was found that made $P$ (Type I error) $=\alpha_{\text {want }}$ to seven decimal places. This was rounded off for Table 1. The Student's $t$ values in Table 1 were found by SAS (1988).

The probability of accepting the population when the true mean has some arbitrary value $\mu$ is evaluated in almost the same way. The region of integration remains exactly the same. The only change is that $\bar{X}_{1}$ and $\bar{X}_{2}$ have mean $\mu$, so the integrand is slightly different. This allows for computation of power curves.

\section{A.2 Simılations with Random $\mathrm{n}_{2}$}

When $n_{2}$ is chosen based on the first sample, instead of being prespecified, the 
integration is more difficult: The dependence of $n_{2}$ on $\bar{x}_{1}$ and $s_{1}{ }^{2}$ must be shown in the limits of integration (A-3) and (A-6), in the normal density of $\bar{r}_{2}$, and in the $\chi^{2}$ density of $\left(n_{2}-1\right) s_{2}{ }^{2}$. Because $n_{2}$ is restricted to integer values, the overall integral is most practically evaluated by breaking up the region of $\bar{x}_{1}$ and $s_{1}{ }^{2}$ into subregions where $n_{2}$ takes its possible values, so that $n_{2}$ is constant within each subregion. This did not seem worth the effort for this report. Therefore, Section 4.2 used simulation instead of integration, as explained here.

As in Section A.1, change the units of $X$ to let the regulatory or QA limit $L$ equal 0 and let $\sigma^{2}=1$, with no loss of generality.

\section{A.2.1 General Procedure}

The samples for Section 4.2 were generated on a Cray X-MP/216. The values of $\bar{x}_{1}$ and $\bar{x}_{2}$ were generated with the IMSL (1987) random number generator RNNOA, which implements the method of Kinderman and Ramage (1976). The values of $s_{1}{ }^{2}$ and $s_{2}{ }^{2}$ were generated with the IMSL subroutine RNCHI, and $s^{2}$, the variance of the pooled sample, was calculated using Equation (A-5) in Section A.1. For a specified $\alpha$, the estimated $P$ (Type I error) and its standard error were calculated as follows. Let $N A 1$ be the number of Monte Carlo runs that result in accepting the population on stage 1. Let $N A 2$ be the number of runs that result in continuing to stage 2 and ultimately accepting the population, and let $N$ denote the total number of Monte Carlo runs. From Equation (1), we have

$P($ Type I error $)=\alpha+P(C 1$ and $A 2)$

which is estimated by $\widehat{p}=\alpha+(N A 2 / N)$. Define the term in parentheses as $\widehat{p}_{2}$, so $\widehat{p}_{2}=\widehat{p}-\alpha$. The standard error of $\widehat{p}$ equals the standard error of $\widehat{p}_{2}$, which equals $\left[\widehat{p}_{2}\left(1-\widehat{p}_{2}\right) / N\right]^{1 / 2}$. This is smaller that the standard error that would result from e-timating $\widehat{p}$ by $(N A 1+N A 2) / N$; that standard error would be $[\widehat{p}(1 \cdots \widehat{p}) / N]^{1 / 2}$.

For computing the power of the test at $\mu \neq L$, the same idea was followed, except that we had 
$P($ accept population $)=P(A 1)+P(C 1$ and $A 2)$.

and $P(A 1)$ was evaluated using the IMSL non-central $t$ function TNDF, with noncentrality parameter $(\mu-L) \sqrt{n_{1}}$.

\section{A.2.2 Computation of Table 2}

Let $a_{0}$ denote the nominal a that yields the desired $P$ (Type I error). Monte Carlo simulations were used to esimate $P$ (Type I error) for various nominal $\alpha$. The method of false position was used to search iteratively for $\alpha_{0}$, with the Monte Carlo sample sizes increasing as a approached $\alpha_{0}$. Monte Carlo sample sizes as large as $2,000,000$ were used at the end of the search. The final estimate was obtained by weighted least squares as follows. The independent variables ( $x$ values) were the set of nominal $\alpha$ 's that had been used during the false-position search. The dependent variables ( $y$ values) were the corresponding set of $P$ (Type I urror) estimates, with known standard errors (denoted $s e$ ). The $y$ values were fitted to a linear function of $x$ by weighted least squares, with weights proportional to $1 / s e^{2}$. Because there was no guarantee that $y$ is a linear function of $x$. values with $x$ far from the final cluster of $x$ 's were not used; the minimum and maximum $x$ used were not allowed to differ from each other by more than about 2 in the second significant digit. The estimate of $\alpha_{0}$ tabulated in Table 2 is the value of $x$ corresponding to a fitted $y$ equal to the desired $P$ (Type I error).

The standardized residuals were examined, based on the assumption that the random number generator worked correctly so the standard deviations of the $y$ 's were known to high accuracy. This examination raised no questions. In particular, the set of 285 standardized residuals was examined together. The correspond to the 285 simulations used to obtain the 36 values in Table 2. The sample skewness and kurtosis were near the theoretical values, and the Shapiro-Wilk test sected normality only at the significance level of 0.16 , not a small enough value to cause concern. The calculations were performed by SAS (1988). 
The confidence interval for $\alpha_{0}$ was found using Fieller's method (see Read, 1983) as follows. First, the data were translated so that the $y$ intercept corresponded to the desired $P$ (Type I error). The curve $y=a+b x$ was fitted to the translated cata. Let $a$ and $b$ denote the values corresponding to the mean values of $y$, and let $\widehat{a}$ and $\widehat{b}$ be the fitted values. Weighted least squares were used because the $y$ 's did not all have the same variances. Because the $y$ intercept corresponds to the desired $P$ (Type I error), the value $\alpha_{0}$ equals $-a / b$. Fieller's method uses the fact that

$\widehat{a}-\alpha_{0} \widehat{b}$

is normal with mean 0 and variance

$\operatorname{var}(\widehat{a})+\alpha_{0}^{2} \operatorname{var}(\widehat{b})-2 \alpha_{0} \operatorname{cov}(\widehat{a}, \widehat{b})$

Therefore, with $95 \%$ confidence $\alpha_{0}$ satisfies

$\left(\widehat{a}-\alpha_{0} \widehat{b}\right)^{2} \leq 1.96^{2}\left[\operatorname{var}(\widehat{a})+\alpha_{0}^{2} \operatorname{var}(\widehat{b})-2 \alpha_{0} \operatorname{cov}(\widehat{a}, \widehat{b})\right]$.

The confidence interval for $\alpha_{0}$ is then found by using the quadratic formula. Actually, for greater numerical stability, the data were also translated in the $x$ direction, so that the $x$ 's were centered and $\operatorname{cov}(\widehat{a}, \widehat{b})$ was therefore zero. The $x$-shift was then added back to $\widehat{\alpha}_{0}$ and to the confidence interval, to produce Table 2 .

For each case in Table 2, the results were compared to the original data plot of $x$ versus $(y \pm 2 s e)$. If there were no data points near the ends of the confidence interval for $\alpha_{0}$, or if either end of the confidence interval differed from $\alpha_{0}$ by more than 2 in the third significant digit, additional Monte Carlo simulations were run at selected values of $x$ and the line was refitted using the original data combined with the additional data. For any Monte Carlo run after the first, the initial seed was set to the final seed from the previous run. In the end, every value in Table 2 was based on fitting at least five data points, and each plot of $x$ versus $(y \pm 2 s e)$ was visually consistent with the slaimed accuracy of the table.

The non-monotonicity of portions of Table 2 is surprising, but no error in the 
above procedure has been found. The confidence bounds for the three $\alpha$ values corresponding to $P$ (Type I error) $=0.10$ and $w=4$ are $(0.07130,0.07149),(0.07098$, $0.07120)$, and $(0.07124,0.07141)$ respectively. This is evidence of non-monotonicity which is not easily explainable by Monte Carlo uncertainty.

The values of $t_{1-\alpha}\left(n_{1}-1\right)$ in Table 2 were found by SAS (1988).

\section{References}

IMSL (1987), User's Manual: STAT/LIBRARY, Version 1.0, Houston: IMSL, Inc.

Kinderman, A. J. and J. G. Ramage (1976), "Computer Generation of Normal Random Variables," Journal of the American Statistical Association, 71, pp. 893-896.

Read, Campbell B. (1983), "Fieller's Theorem," Encyclopedia of Statistical Sciences, Vol. 3, New York: John Wiley \& Sons.

SAS (1988), SAS Language Guide for Personal Computers, Release 6.03 Edition. Cary, NC: SAS Institute, Inc. 

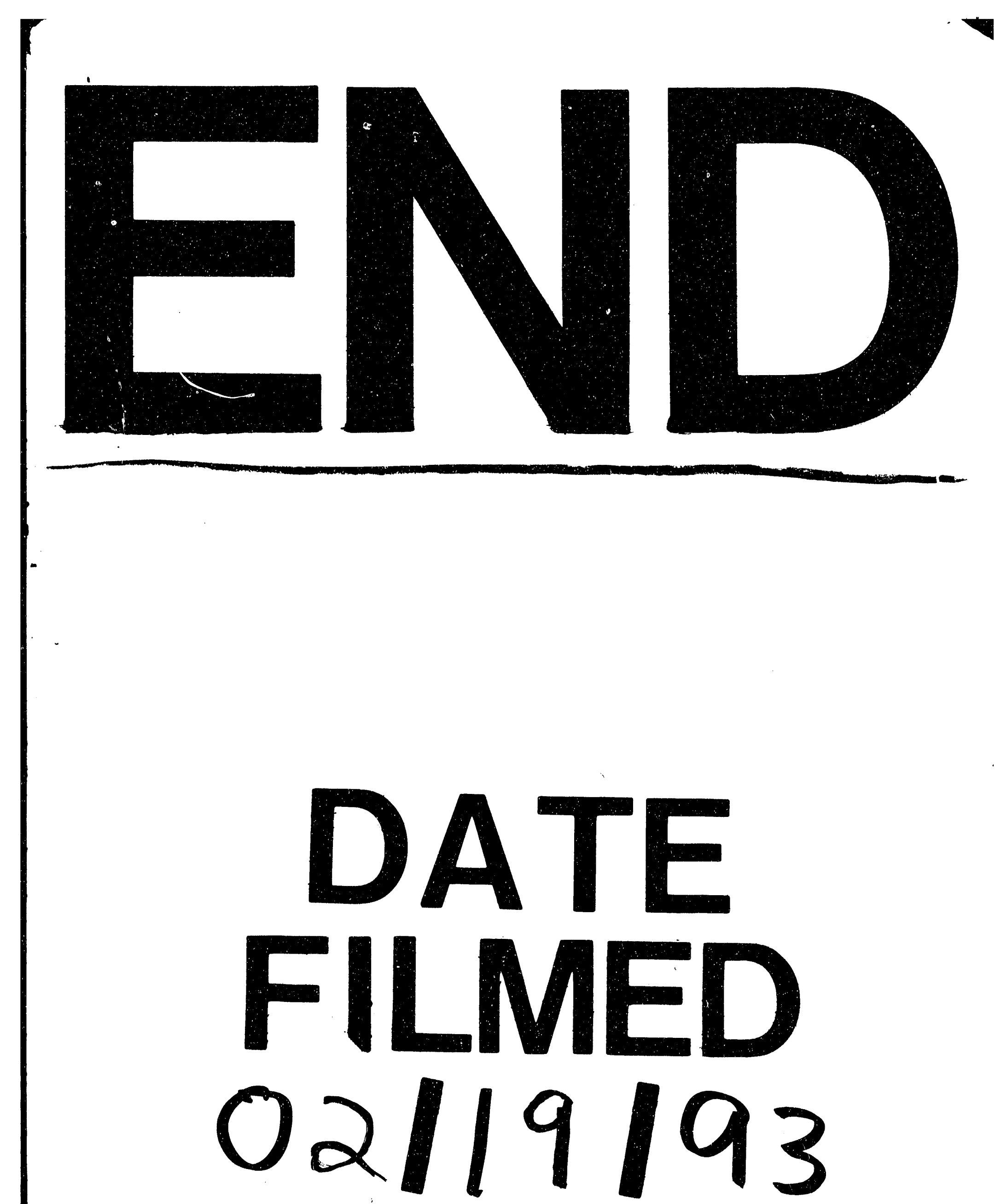


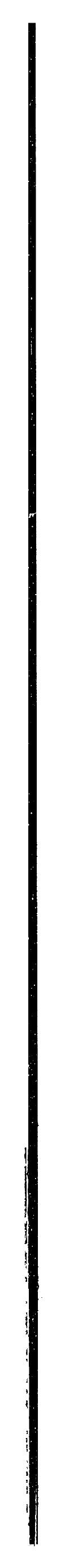

